\title{
KINETIC ANALYSIS OF THE ABNORMAL FLUORIMETRIC TITRATION BEHAVIOUR OF NAPHTHYLAMINES
}

\author{
Fnedrıch HAFNER, Jörg WÖRNER, Ulrich STEINER and Manfred HAUSER \\ Instutut für Physikalısche Chemie der Universiät Stuttgart, D-7000 Stuttgart 80, West Germany
}

\begin{abstract}
The fluorimetric titration behaviour of naphthylamines is abnormal in that the sum of the reduced quantum yrelds of conjugated acid and base passes through a minımum. Based on fluorescence quantum yield and lifetime measurements, we explain this behaviour by two independent mechanisms: A diabatic quenching of the excited base by protons and a decrease of the excited cation's protoly tic dissociation in the hyperacidic region. Though apparently independent processes, theu rate constants can be shown to be related to each other by a quasi-thermodynamical equation.
\end{abstract}

\section{Introduction}

In the investigation of the behaviour of acid-base reactions in the excited singlet state, the application of stationary and time-dependent fluorescence measurements is well established [1]. Normally the sum of the reduced quantum yields of the acidic form $\mathrm{AH}^{+}$and the neutral form $\mathrm{A}$ is unity,

$\Phi_{\mathrm{AH}^{+}} / \Phi_{\mathrm{AH}^{+}}^{\max }+\Phi_{\mathrm{A}} / \Phi_{\mathrm{A}}^{\max }=1$,

whuch is a consequence of adiabaticity ("conservation of excitation") in the excited-state protolytic reactions. One famous exception to this relation is the fluorescence change of naphthylamme and its derivatives, first investıgated by Forster $[2,3]$ and Schulman [4]. Whereas in systems obeying eq. (1) the fluorescence decrease of the basic component is accompanied by a concomitant increase of the acid component, in the case of the naphthylamines the fluorescence change of either component occurs in its own region of $p H$ or $H_{0}$, respectively.

As an explanation those authors suggested that a non-fluorescent intermediate should be involved in the proton-transfer reaction, opening a channel of effective radiationless deactivation and thus causing a drastic decrease in the sum of reduced quantum yields in moderately acidic solutions. As possible nonfluorescent intermediate, an exciplex between hy- dronium ion and naphthylamine $[3,4]$ or a ringprotonated species [3], as suggested by Weller, were considered.

Tsutsumı and Shizuka (TS) [5] analyzed the quantum yield and lifetime of the amine fluorescence in the $p H$ region $1 \leqslant p H \leqslant 3$ in terms of proton-induced quenching, which was shown to dominate the adiabatic protonation of the amine. These authors were not interested in the increase of the fluorescence intensity of the protonated form $\mathrm{AH}^{+}$in the hyperacidic region $\left(-3 \geqslant H_{0} \geqslant-6\right)$.

Schulman and Surgeon [6] recently reinvestigated the fluorescence change of naphthylamines. According to their measurements the lifetime of the excited $\mathrm{AH}^{+}$is independent of the acid concentration. Therefore, to explain the change of the cationic fluorescence quantum yield, they had to assume a static quenching mechanism for which they suggested the following ground-state hydration equilubrium:

$\left(\mathrm{AH}^{+}\right)\left(\mathrm{H}_{2} \mathrm{O}\right)_{n} \rightleftharpoons \mathrm{AH}^{+}+\imath \mathrm{H}_{2} \mathrm{O}$,

where only the unhydrated species (present in concentrated acid) was assumed to be fluorescent.

Measurements in our laboratory have shown that the lifetime of the excited $\mathrm{AH}^{+}$changes with acid concentration in the same way as the quantum yield. We are able to explain the quenching of the neutral form as well as the increase of the fluorescence in- 
tensity of the cationic species in the highly acidic region. Furthemore we can show that the cationic and neutral base fluorescences, though kinetically apparently independent, are related to each other by a quasi-thermodynamic relation.

\section{Experimental}

1-naphthylamıne (Merck, reagent grade) was used without further purification 2-naphthylamine (Merck) was purified via its liydrochloride and by subsequent sublimation. Reagent-grade $\mathrm{H}_{2} \mathrm{SO}_{4}$ (Merck) and deionized and doubly distulled water were used, the actual acid contents deternuned by titration. The amine concentrations were $2 \times 10^{-4} \mathrm{M}$ ( 1 -naphthylamıne) and $8 \times \mathrm{i0}^{-4} \mathrm{M}$ (2-naph thylamine). The investigations were carried out with solutions showing cationic absorption spectra. Only freshly prepared solutions were investigated in order to avold chemical reactions of the naphthylammes.
The measurements were made on air-saturated solutions, since control experiments with degassed solutions showed that oxygen does not influence the quantum yields and lifetımes.

Fluorescence spectra were recorded with a HitachlPerkin-Elmer (MPF-3L) spectrophotometer. Slit wid ths smaller than $8 \mathrm{~nm}$ were used. For determination of absolute fluorescence quantum yrelds indole in ethanol $\left(\Phi_{F}=036[7]\right)$ was used as a quantum standard for the cationic fluorescence and quinine bisulfate in $0.1 \mathrm{~N} \mathrm{H}_{2} \mathrm{SO}_{4}\left(\Phi_{\mathrm{F}}=0.54\right.$ [8] ) for the amine fluorescence. Lifetume measurements were performed on a single photon counting apparatus (Ortec), with a half wid th of the exciting flash of 2.5 ns.

\section{Results and discussion}

Quantum yields and fluorescence decay constants of 1-and 2-naphthylamine are given in tables 1 and 2 for various concentrations of $\mathrm{H}_{2} \mathrm{SO}_{4}$ where the quan-

Table 1

I Iuorescence quantum yiclds and decay constants of l-naphthylamine (free base $\mathrm{RNH}_{2}$ and cation $\mathrm{RNH}_{3}^{+}$) at varıous acıd concentrations

\begin{tabular}{|c|c|c|c|c|c|c|c|}
\hline \multicolumn{4}{|l|}{$\mathrm{RNH}_{3}^{+}$} & \multicolumn{4}{|l|}{$\mathrm{RNH}_{2}$} \\
\hline $\begin{array}{l}c_{\mathrm{H}_{2} \mathrm{SO}_{4}} \\
(\mathrm{~mol} / \mathrm{R})\end{array}$ & ${ }^{\infty} \mathrm{AH}^{+}$ & ${ }_{\left(10^{6} \mathrm{~s}^{-1}\right)}$ & $\begin{array}{l}k_{1} \\
\left(10^{6} s^{-1}\right)\end{array}$ & $\underset{(\mathrm{mol} / \mathrm{R})}{c_{\mathrm{H}_{2}} \mathrm{SO}_{4}}$ & $\Phi_{A}$ & $\begin{array}{l}\lambda_{2} \\
\left(10^{6} s^{-1}\right)\end{array}$ & $\begin{array}{l}k_{\mathrm{f}}^{\prime} \\
\left(10^{6} \mathrm{~s}^{-1}\right)\end{array}$ \\
\hline 99 & 024 & 20 & 48 & 0001 & 028 & 56 & 156 \\
\hline 86 & 020 & 23 & 46 & 0005 & 015 & 109 & 16.4 \\
\hline 77 & 0.15 & 34 & 51 & 0009 & $010_{2}$ & 160 & 163 \\
\hline 71 & $012_{5}$ & 36 & 4.5 & 0013 & 0079 & 200 & 158 \\
\hline 57 & 0072 & $6 \div$ & 46 & 0018 & 0061 & 256 & 156 \\
\hline 51 & 0046 & 100 & 46 & 0031 & 0041 & 392 & 160 \\
\hline
\end{tabular}

Table 2

Fluorescence quantum yields and decay constants of 2-naphthylamine (free base $\mathrm{RNH}_{2}$ and cation $\mathrm{RNH}_{3}^{+}$) at various acid concentrations

\begin{tabular}{|c|c|c|c|c|c|c|c|}
\hline \multicolumn{4}{|l|}{$\mathrm{RNH}_{3}^{+}$} & \multicolumn{4}{|l|}{$\mathrm{RNH}_{2}$} \\
\hline${ }_{(\mathrm{mol} / \mathrm{e})}^{c_{\mathrm{H}_{2}} \mathrm{SO}_{4}}$ & $\Phi_{\mathrm{AH}^{+}}$ & $\begin{array}{l}\lambda_{2} \\
\left(10^{6} \mathrm{~s}^{-1}\right)\end{array}$ & $\begin{array}{l}k_{\mathrm{f}} \\
\left(10^{6} \mathrm{~s}^{-1}\right)\end{array}$ & $\begin{array}{c}c_{\mathrm{H}_{2} \mathrm{SO}_{4}} \\
(\mathrm{~mol} / \mathrm{q})\end{array}$ & $\mathbf{\Phi}_{\mathbf{A}}$ & $\begin{array}{l}\lambda_{2} \\
\left(10^{6} \mathrm{~s}^{-1}\right)\end{array}$ & $\left(10^{6} s^{-!}\right)$ \\
\hline 102 & 0060 & 40.1 & 240 & 0.001 & 0.39 & 570 & 222 \\
\hline 9.7 & 0059 & 422 & 249 & 0103 & 027 & 83.1 & 224 \\
\hline 87 & 0042 & 59.0 & 2.48 & 0205 & 020 & 108 & 21.6 \\
\hline 755 & 0019 & 132 & 251 & 0.423 & 013 & 176 & 228 \\
\hline 67 & $0010_{5}$ & 240 & 252 & 063 & 0092 & 245 & 225 \\
\hline 6.0 & $0006_{4}$ & 395 & 253 & 086 & 0065 & 336 & 21.8 \\
\hline
\end{tabular}


tum yields are high enough to allow accurate lifetıme measurements In the intermediate acidity range only the quantum yields were determined. The results obtained for these are in fair agreement with TS (see fig. 1 of ref. [5]).

We first discuss the fluorescence behaviour of the neutral compounds, which at $p H \leqslant 3$ is observed on excitation of the cationic forms. As already described by TS the corresponding fluorescence decay curves, as measured by single photon countung technique. can be fitted by convolution of the exciting pulse with the following fluorescence response function $I_{\mathrm{A}}(t)$ to $\delta$-pulse excitation

$I_{\mathrm{A}}(t) \propto\left(\mathrm{e}^{-\lambda_{1} t}-\mathrm{e}^{-\lambda_{2} t}\right)$.

For 1- and 2-naphthylamine the slow time constants, describing the decay at long tumes, can be determined with good accuracy. They are given in tables 1 and 2 for various acid concentrations. In each case the product of decay constant and quantum yreld results in a constant value for the rate constant of fluorescence $\left(k_{\mathrm{f}}^{\prime}\right)$, independent of the $p H$ of the solution.

The fast time constant could be determined by single photon technique with reliable accuracy for 1-naphthylamine only It was found to be $8.5 \times 10^{8}$ $s^{-1}$ at $p H 27$. In the case of 2-naphthylamine the tume resolution of the single photon apparatus was not sufficient to resolve the fast tume constant, which, according to eq. (3), determines the rise of the fluorescence of $A$. If the excited $A$ is formed from the fluorescent cationic species the fast decay of $\mathrm{AH}^{+}$ must be identical with the rise of A-fluorescence. Unfortunately the cationic fluorescence, though detectable whenever $\mathrm{AH}^{+}$is excited, is too weak for accurate lifetime measurements in the acidity region where lifet tme measurements of the neutral base fluorescence are feasible. However, using the rate constant of fluorescence of $\mathrm{AH}^{+}\left(4.8 \times 10^{6} \mathrm{~s}^{-1}\right.$ for 1-naphthylamine and $25 \times 10^{6} \mathrm{~s}^{-1}$ for 2 -naphthylamine) measured in the hyperacidic region where the quantum yreld of $\mathrm{AH}^{+}$is high, and using the quantum yield of $\mathrm{AH}^{+}$at $p H 3$ (0.006 for 1-naphthylamine and 0.0007 for 2-naphthylamine), fluorescence decay constants at this $p H$ are calculated to be $8.0 \times 10^{8}$ $\mathrm{s}^{-1}$, respectively. The value for 1 -naphthylamine is in good agreement with the fast tume constant obtained from the single photon counting measurement on the amine fluorescence. The rate constant for 2- naphthylamine is indeed of an order of magnitude not resolvable by this experimental technique and so far in accord with the experimental findings. We consider these results as evidence that the fluorescent species $\left(\mathrm{AH}^{+}\right)^{*}$ is the direct precursor of $\mathrm{A}^{*}$, when formed via excitation of $\mathrm{AH}^{+}$.

This conclusion is in direct contradiction to the paper of Schulman and Surgeon [6] who attributed the formation of $A^{*}$ to the dissociation of a nonfluorescent hydrated cation and assumed the cationic fluorescence to be emitted by a non-hydrated cation. These conclusions are based, however, on an erroneous experumental result concerning the acidity dependence of the lifetime of the cationic fluorescence, which is disproved by our measurements.

If, as we conclude from our results, there is only one kind of excited cation, the decrease of lifetime and quantum yield of the neutral fluorescence with increasing acid concentration cannot be expiained by an adiabatic protonation of $A^{*}$, since this would be indicated by a corresponding increase of the cationic fluorescence intensity. At a $p H$, however, where the neutral fluorescence has dropped to one percent of its initial value, the cationic fluorescence has increased to less than $1 \%$ of its maximal value (cf. also fig. 1 of TS [5]). In agreement with TS we conclude therefore that there is a drabatic quenching process by protons, the rate constant $\left(k_{\mathrm{q}}^{\prime}\right)$ of which exceeds by far the rate constant of the adiabatic association process $\left(k_{2}\right)$. In the following we make use of the kinetic scheme of TS, adopting the same symbols for the rate constants. We show, that with some modifications this scheme can account for all the features of the abnormal fluonmetric titration curves (see scheme I)

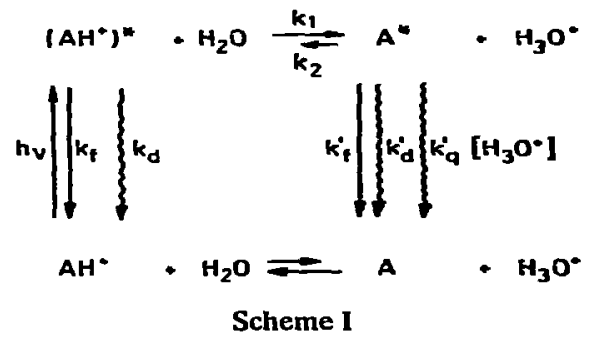

For the $p H$-region where lifetume measurements of $A^{*}$ are feasible (see tables 1 and 2), the results described so far can be summarized by the relations: 


$$
\begin{gathered}
k_{1} \gg k_{\mathrm{q}}^{\prime}\left[\mathrm{H}_{3} \mathrm{O}^{+}\right] \gg k_{2}\left[\mathrm{H}_{3} \mathrm{O}^{+}\right], k_{1} \gg k_{0} \approx k_{0}^{\prime} \\
\left(k_{0}=k_{\mathrm{f}}+k_{\mathrm{d}}, k_{0}^{\prime}=k_{\mathrm{t}}^{\prime}+k_{\mathrm{d}}^{\prime}\right) .
\end{gathered}
$$

$W_{1}$ th these conditions the following expression can be derived for the fluorescence quantum yield of $A$ (cf. eq. (2) of TS [5])

$\Phi_{\mathrm{A}}=\Phi_{\mathrm{A}}^{\max } k_{0}^{\prime} /\left(k_{0}^{\prime}+k_{\mathrm{q}}^{\prime}\left[\mathrm{H}_{3} \mathrm{O}^{+}\right]\right)$.

For the two rate constants of fluorescence of $A$ (and $\mathrm{AH}^{+}$) one obtains

$$
\begin{aligned}
\lambda_{1,2} & =\frac{1}{2}\left\{k_{0}+k_{1}+k_{0}^{\prime}+\left(k_{\mathrm{q}}^{\prime}+k_{2}\right)\left[\mathrm{H}_{3} \mathrm{O}^{+}\right]\right. \\
& \pm\left(k_{0}+k_{1}-k_{0}^{\prime}-\left(k_{\mathrm{q}}^{\prime}+k_{2}\right)\left[\mathrm{H}_{3} \mathrm{O}^{+}\right]\right) \\
& \times\left\{1+4 k_{1} k_{2}\left[\mathrm{H}_{3} \mathrm{O}^{+}\right]\right. \\
& \left.\left.\times\left(k_{0}+k_{1}-k_{0}^{\prime}-\left(k_{\mathrm{q}}^{\prime}+k_{2}\right)\left[\mathrm{H}_{3} \mathrm{O}^{+}\right]\right)^{-2}\right\}^{1 / 2}\right\}
\end{aligned}
$$

which, under the condition (4) valıd at small values of $\left[\mathrm{H}_{3} \mathrm{O}^{+}\right]$and using the approximation $(1+x)^{1 / 2}$ $\approx 1+x / 2($ for $x<1)$, can be simplified to

$\lambda_{1}=k_{0}+k_{1}+k_{2}\left[\mathrm{H}_{3} \mathrm{O}^{+}\right]$,

$\lambda_{2}=k_{0}^{\prime}+k_{\mathrm{q}}^{\prime}\left[\mathrm{H}_{3} \mathrm{O}^{+}\right]$.

From (5) and (8) it follows immediately that for the slow tume constant $\lambda_{2}$, evaluated from the single photon experments, the following relation must hold

$\lambda_{2} \Phi_{\mathrm{A}}=k_{0}^{\prime} \Phi_{\mathrm{A}}^{\mathrm{max}}=k_{\mathrm{f}}^{\prime}=$ const.,

which is confirmed experimentally (see tables 1 and 2). Both equations (5) and (8) are Stern-Volmer relations, yielding linear plots in the limit of low $\mathrm{H}^{+}$concentration. From either of these $k_{q}^{\prime}$ can be calculated. Our values of $58 \times 10^{9} \mathrm{M}^{-1} \mathrm{~s}^{-1}$ and $1.9 \times 10^{8}$ $M^{-1} s^{-1}$ for 1- and 2-naphthylamine, respectively, are in fair agreement with those obtained by TS As for $k_{2}$, it is clear from eqs. (7) and (8) that under the conditions, indicated in eq. (4), information can be only obtained from the fast time constant $\lambda_{1}$ This requires, however, that $\lambda_{1}$ is measured with an accuracy of better than $1 \%$ which, in our opinion is outside the scope of a nanosecond technique, since $\lambda_{1} \geqslant 10^{9} \mathrm{~s}^{-1}$. Therefore one cannot obtain kinetically determined $p K_{\mathrm{a}}^{*}$ values for the naphthylammes.

In the hyperacidic region $\left(5<c_{\mathrm{H}_{2} \mathrm{SO}_{4}}<10 \mathrm{~mol} / \mathrm{l}\right.$ corresponding to approximately $-2.5>H_{0}>-6$ ) the fluorescence of $\mathrm{AH}^{+}$increases strongly with the acid concentration (see tables 1 and 2 ) and lifetime measurements can be performed by the single photon technıque. The fluorescence decay curves are monoexponentral and the product of decay constant and quantum yield is constant, indicating that $k_{\mathrm{f}}$ is constant This justifies our assumption that in the region where the lifetine of $\left(\mathrm{AH}^{+}\right)^{*}$ is too short to be determined experimentally, $k_{\mathrm{f}}$ is still unchanged, allowing the determination of the decay time from the quantum yield.

Based on our experimental results, we explain the increase of the cationic fluorescence in the strongly acidic region as follows The single-exponential decay of this fluorescence is in accord with the assumption $k_{2} \ll k_{\mathrm{q}}^{\prime}$ [cf. eq. (4)]. Therefore the kinetic problem is that of a single, directly excited species, in which case we have

$\Phi_{\mathrm{AH}^{+}}=k_{\mathrm{f}} /\left(k_{\mathrm{f}}+k_{\mathrm{d}}+k_{1}\right)$.

Since $k_{\mathrm{f}}$ is constant, as demonstrated in tables 1 and 2 , and since for low proton concentrations, where

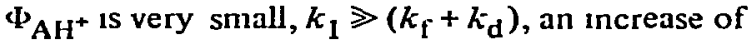

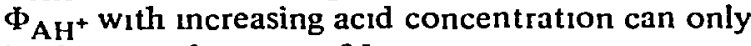
be due to a decrease of $k_{1}$.

To understand this behaviour it is important to note, that in the hyperacidic region, where the increase of the cationic fluorescence begins, the activlty of the proton acceptor water becomes markedly reduced. We do not give a mechanistic interpretation of the decrease of $k_{1}$ here, but instead point out that a quasi-thermodynamic relation exists between $k_{i}$ and the quenching constant $k_{\mathrm{q}}^{\prime}$. This rate parameter, again, is not a true constant, but increases as the acidity of the solution increases. This was also observed by TS.

Since the character of the solvent is changed when proceeding from dilute aqueous to highly acıdic solutions, one cannot expect the rates of protonation or quenching of $\mathrm{A}^{*}$ to be described correctly by $k_{2}\left[\mathrm{H}_{3} \mathrm{O}^{+}\right]\left[\mathrm{A}^{*}\right]$ or $k_{\mathrm{q}}^{\prime}\left[\mathrm{H}_{3} \mathrm{O}^{+}\right]\left[\mathrm{A}^{*}\right]$, respectively, if $k_{2}$ and $k_{\mathrm{q}}^{\prime}$ are regarded as true rate constants. Therefore we generalıze these expressions by introducing the $\left[\mathrm{H}_{3} \mathrm{O}^{+}\right]$-dependent rate "constants" $\widetilde{k}_{2}$ and $\widetilde{k}_{q}^{\prime}$, with the only assumption that their ratio is constant:

$\widetilde{k}_{2} / \widetilde{k}_{\mathrm{q}}^{\prime}=c$. 
The rate parameter $\widetilde{k}_{q}^{\prime}$ can be calculated from the quantum yield of the free base $\Phi_{A}$ by use of eq. (5):

$\widetilde{k}_{\mathrm{q}}^{\prime}=\left(\Phi_{\mathrm{A}}^{\max } / \Phi_{\mathrm{A}}-1\right) k_{0}^{\prime} /\left[\mathrm{H}_{3} \mathrm{O}^{+}\right]$.

We also use $\widetilde{k}_{1}$ instead of $k_{1}$ in order to express the $\left[\mathrm{H}_{3} \mathrm{O}^{+}\right]$-dependence of this rate constant. It can be calculated by the equation.

$\widetilde{k}_{1}=k_{0}\left(\Phi_{\mathrm{AH}^{+}}^{\mathrm{max}} / \Phi_{\mathrm{AH}^{+}}-1\right)$.

We now consider the hypothetical case that all rate constants of scheme I are zero except $\widetilde{k}_{1}$ and $\widetilde{k}_{2}$.

Then a true thermodynamic equilibrium between $A^{*}$ and $\left(\mathrm{AH}^{+}\right)^{*}$ would be established in the excited state, which in the hyperacidic region would be determined by the $p K_{a}^{*}$ and the Hammett acidity function $H_{0}$ [9] :

$\log \left[\mathrm{A}^{*} /\left(\mathrm{AH}^{+}\right)^{*}\right]=H_{0}-\vec{p} K_{\mathrm{a}}^{*}$,

if we assume that the $H_{0}$ function, valid for the ground state, can also be applied to the excited state.
On the other hand, for kinetic reasons, the following equation would hold:

$$
\left[\mathrm{A}^{*}\right] /\left[\left(\mathrm{AH}^{+}\right)^{*}\right]=\widetilde{k}_{1} / \widetilde{k}_{2}\left[\mathrm{H}_{3} \mathrm{O}^{+}\right]=\widetilde{k}_{1} c / \widetilde{k}_{\mathrm{q}}^{*}\left[\mathrm{H}_{3} \mathrm{O}^{+}\right] \text {. }
$$

Hence from eqs. (14) and (15) we obtain:

$\log \widetilde{k}_{1} \log \left(\widetilde{k}_{q}^{\prime}\left[\mathrm{H}_{3} \mathrm{O}^{+}\right]\right)=H_{0}-p K_{\mathrm{a}}^{*}-\log c$.

Substituting $\widetilde{k}_{1}$ and $\widetilde{k}_{q}^{\prime}$ by the corresponding quantum yield expressions (12) and (13) leads to:

$$
\begin{gathered}
\log \left(\Phi_{\mathrm{AH}^{+}}^{\max } / \Phi_{\mathrm{AH}^{+}}-1\right)-\log \left(\Phi_{\mathrm{A}}^{\max } / \Phi_{\mathrm{A}}-1\right) \\
=H_{0}-p K_{\mathrm{a}}^{*}-\log \left(\kappa_{0} / k_{0}^{\prime}\right)-\log c .
\end{gathered}
$$

Thus we have arrived at a quast-thermcdynamic relation between functions of the fluorescence quantum yields of excited acid and base and the acidity function $H_{0}$, though different mechanisms affect these quantum yields. The plots of the left hand side of eq. (17) versus $H_{0}$ are shown in fig. 1 . For both amines

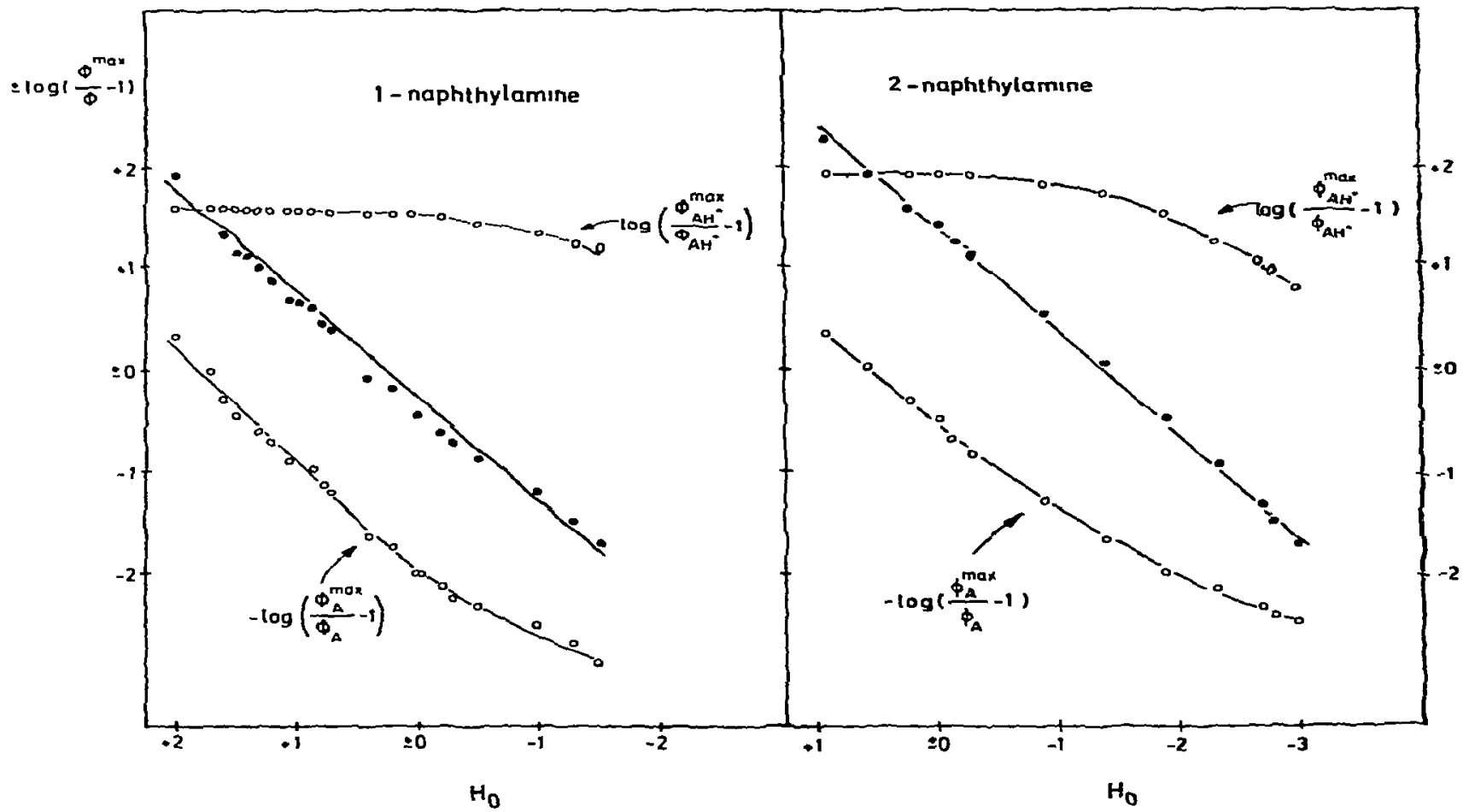

Fig. 1. Plot of left-hand side terms of eq. (17) versus Hammett acidity function $\mathrm{H}_{0}$ (conversion of $\mathrm{H}_{2} \mathrm{SO}_{4}$-concentration to $\mathrm{EL}_{0}$ accordung to ref. [10]). Open curcles: undividual contributsons of cationic and neutral amine fluorescence. Full circles: difference of the respective contributions 
straight lines are obtained with almost theoretical slopes ( 102 ).

\section{Conclusions}

Our experimental results on the acidity dependence of the fluorescence quantum yield and lifetıme of 1 and 2-naphthylamine and their respective cations lead us to the following explanation of the exceptional fluorescence beliaviour of these compounds in the spectral turnover region: The fliorescence of the excited bases, formed upon adiabatic dissociation of the excited cations, decreases (equally in quantum yield and lifetime) with increasing acidity dice to a diabatic quenching of the excited bases by protons The fluorescence of the excited cations increases with acidity in the hyperacidic region (equally in quantum yield and If fetime) not because of a reprotonation of the excited bases, but because of a decrease of the protoly'tlc dissociatıon rate constant $\left(k_{1}\right)$. The latter has to be explained by a decreasing activity of water, the proton-accepting species in the system. The adrabatic reprotonation of the excited base $\left(k_{2}\right)$ is neghgible compared to the diabatic quenching by protons $\left(k_{\mathrm{q}}^{\prime}\right)^{\ddagger}$. A quasi-thermodynamic relation between $k_{1}$ and $k_{q}^{\prime}$ has been established, even though the corresponding reactions do not compose a chemical equilibrium.

\footnotetext{
F For cases where $k_{\mathrm{q}}^{\prime}$ and $k_{2}$ are comparable sce ref [11].
}

\section{Acknowledgement}

We thank Professor Albert Weller for valuable suggestrons and the Deutsche Forschungsgemeinschaft for financial support.

\section{References}

[1] W.R Ware, in. Creation and detection of the excited state, Vol 1A, ed A. Lamola (Dekker, New York, 1971) p 213, E van der Donckt, Progr Reaction Kinetics 5 (1970) 273

(2) Th rörster, Z. Elektrochem. 54 (1950) 531.

[3] Th Forster, Chem Phys. Letters 17 (1972) 309

[4] S G. Schulman and P Liedke, Z. Physik Chem NF 84 (1973) 317.

[5] K Tsutsumi and $H$ Shrzuka, Chem Phys. Letters 52 (1977) 485

[6] S G Schulman and R.J Sturgeon, ] Am Chem Soc 99.(1977) 7209

[7] J.B. Berlman, Handbook of nuorescence spectra of aromatic molccules, 2nd Ed (Academic Press, New York, 1971).

[8] E Lippert, W Nagele, I Seybold-Blankenstein, U Staiger and W. Voss, $Z$ Anal Chem 170 (1959) 1

[9] C H Rochester, Acıdity functions (Academic Press, Neu York, 1970)

[10] K N Bascombe and R P Bell, J. Chem Soc. (1959) 1096

[11] K. Tsutsumı and H. Shrzuka, Z. Physik Chem NF, to be published. 